\title{
Perfil epidemiológico da dengue e Zika vírus durante a pandemia da Covid-19 em
}

\section{Minas Gerais}

\author{
Epidemiological profile of dengue and Zika virus during the pandemic of Covid-19 in Minas Gerais \\ Perfil epidemiológico del virus del dengue y del Zika durante la pandemia de Covid-19 en Minas
}

Gerais

Recebido: 12/11/2021 | Revisado: 20/11/2021 | Aceito: 25/11/2021 | Publicado: 07/12/2021

\author{
Caíque Olegário Diniz e Magalhães \\ ORCID: https://orcid.org/0000-0001-7440-5463 \\ Universidade Estadual de Minas Gerais, Brasil \\ Universidade Federal Vales do Jequitinhonha e Mucurí, Brasil \\ E-mail: caique-959@ hotmail.com \\ Talita Emanuela Domingues \\ ORCID: https://orcid.org/0000-0001-7282-6491 \\ Universidade Estadual de Minas Gerais, Brasil \\ Universidade Federal Vales do Jequitinhonha e Mucurí, Brasil \\ E-mail:talita.ed@gmail.com \\ José Vitor Vieira Salgado \\ ORCID: https://orcid.org/0000-0002-5205-9341 \\ Universidade Estadual de Minas Gerais, Brasil \\ E-mail: jose.salgado@uemg.br \\ Rayssa Nogueira Rodrigues \\ ORCID: https://orcid.org/0000-0002-4772-4968 \\ Universidade Federal de Viçosa, Brasil \\ E-mail: rayssa.machado@ufv.br
}

\begin{abstract}
Resumo
Devido ao clima predominantemente tropical, o Brasil apresenta alta incidência de arboviroses. Dentre elas destacamse aquelas transmitidas pelo Aedes aegypti, principal vetor para a dengue e o Zika. No entanto, diante da pandemia causada pela Covid-19, a situação no Brasil pode ter mudado, no que se refere ao perfil de notificação e do controle dessas patologias. A fim de verificar tal cenário, realizou-se um estudo ecológico, descritivo, período de 2019 a 2020. Os dados foram obtidos por meio do Sistema de Informações de Agravos de Notificação (SINAN) e o Sistema de Informações sobre Mortalidade (SIM), disponibilizados pelo Departamento de Informática do Sistema Único de Saúde (DATASUS)/Plataforma de Informações em Saúde (TABNET). Os resultados mostraram que no estado de Minas Gerias no ano de 2020 foram confirmados apenas $8,7 \%(\mathrm{n}=85.356)$ casos de dengue e $34 \%$ $(\mathrm{n}=1.350)$ de Zika, em 2019 esse número chegou a 31,0\% ( $\mathrm{n}=478.898)$ casos de dengue e 45,3\% (n=3.228) nos casos de Zika, ou seja, houve uma redução de $82,17 \%$ na notificação em 2020 da dengue e de 58,17\% nos casos de Zika. Esses dados sugerem que esforços para conter a entidade emergente Covid-19 podem inviabilizar a notificação e o diagnóstico efetivo das citadas patologias. Os sintomas semelhantes entre doenças também podem contribuir para tal cenário. Além disso, acredita-se que o temor relacionado à infecção pela Covid-19 tem dificultado a notificação e retardado o diagnóstico. Conclui-se que há necessidade de desenvolvimento de políticas que incentivem a notificação, o controle do patógeno, a educação da população e medidas de saúde em função do diagnóstico precoce e o monitoramento necessários e preditivo.
\end{abstract}

Palavras-chave: Dengue; Zika Vírus; Covid-19; Epidemiologia.

\begin{abstract}
Due to the predominantly tropical climate, Brazil has a high incidence of arboviruses. Among them stand out those transmitted by Aedes aegypti, the main vector for dengue and Zika. However, given the pandemic caused by Covid19, the situation in Brazil may have changed regarding the effectiveness of notification and control of these pathologies. In order to verify such a scenario, an ecological, descriptive study was carried out, from 2019 to 2020. The Notifiable Diseases Information System (SINAN) and the Mortality Information System (SIM) are made available by the Department of Informatics of the Unified Health System (DATASUS) through the Health Information Platform (TABNET). The results showed that in the state of Minas Gerias, in 2020 only $8,7 \%(n=85,356)$ cases of dengue and 34\% ( $n=1,350)$ of Zika were confirmed, in 2019 this number reached $(n=478,898)$ cases of dengue and $45,3 \%(n=3,228)$ in cases of Zika, i.e., there was a reduction of $82.17 \%$ in dengue notification in 2020 and
\end{abstract}


$58.17 \%$ in cases of Zika Virus. These data suggest that efforts to contain the emerging entity Covid-19 may make it impossible to notify and effectively diagnose the aforementioned pathologies. Similar symptoms among diseases may also contribute to such a scenario. In addition, it is believed that the fear related to Covid-19 infection has made it difficult to report and delay the diagnosis. It is concluded that there is a need for the development of policies that encourage notification, control of the pathogen, population education and health measures due to early diagnosis and monitoring of necessary and predictive.

Keywords: Dengue; Zika Virus; Covid-19; Epidemiology.

\begin{abstract}
Resumen
Debido al clima predominantemente tropical, Brasil tiene una alta incidencia de arbovirus. Entre ellos destacan los transmitidos por el Aedes aegypti, principal vector del dengue y el Zika. Sin embargo, dada la pandemia causada por el Covid-19, la situación en Brasil puede haber cambiado con respecto a la efectividad de la notificación y el control de estas patologías. Para verificar tal escenario, se realizó un estudio ecológico, descriptivo, de 2019 a 2020. El Sistema de Información de Enfermedades de Declaración Obligatoria (SINAN) y el Sistema de Información de Mortalidad (SIM) son disponibles por el Departamento de Informática del Sistema Único de Salud (DATASUS) a través de la Plataforma de Información de Salud (TABNET). Los resultados mostraron que en el estado de Minas Gerias, en 2020 solo 8,7\% ( $\mathrm{n}=85.356)$ se confirmaron casos de dengue y 34\% ( $\mathrm{n}=1.350)$ de Zika, en 2019 este número alcanzó 31\% ( $\mathrm{n}=478.898)$ casos de dengue y 45,3\% $(\mathrm{n}=3.228)$ en casos de Zika, es decir, hubo una reducción de $82,17 \%$ en la notificación de dengue en 2020 y de 58,17\% en casos de virus del Zika. Estos datos sugieren que los esfuerzos para contener a la entidad emergente Covid-19 pueden hacer imposible notificar y diagnosticar eficazmente las patologías antes mencionadas. Síntomas similares entre las enfermedades también pueden contribuir a tal escenario. Además, se cree que el miedo relacionado con la infección por Covid-19 ha dificultado la denuncia y retraso del diagnóstico. Se concluye que existe la necesidad de elaborar políticas que fomenten la notificación, el control del patógeno, la educación de la población y las medidas sanitarias debidas al diagnóstico precoz y al seguimiento necesarios y predictivo.
\end{abstract}

Palabras clave: Dengue; Zika Virus; Covid-19; Epidemiología.

\title{
1. Introdução
}

Desde março de 2020, o Brasil enfrenta uma pandemia da Covid-19 e, desde a confirmação dos primeiros casos, observou-se uma diminuição dos registros de casos prováveis e óbitos de dengue e Zika. Esta diminuição pode ser consequência de uma subnotificação ou atraso nas notificações das arboviroses associadas a mobilização das equipes de vigilância e assistência para o enfrentamento da pandemia e ao receio da população em procurar atendimento em uma unidade de saúde (Nascimento, et al., 2021).

O Brasil é formado por uma grande extensão terrestre de florestas Amazônicas bem distribuídas com concentração principalmente na região norte, mas as regiões, leste, sudeste e litoral do país também apresentando clima tropical predominante, logo, um local adequado para a existência do vetor e assim episódios de arboviroses (Pereira et al., 2019; WHO, 2020).

Os vírus da dengue e do Zika são flavivírus intimamente semelhantes no que se refere ao ciclo de transmissão, à distribuição por trópicos e subtrópicos e às manifestações das doenças, incluindo febre, erupção cutânea, mialgia e artralgia (Sharp et al., 2019). O Aedes aegypti é o principal vetor para a dengue e o Zika, portanto, as medidas de vigilância geralmente se concentram na detecção e monitoramento de populações deste mosquito altamente antropofílico (Eiras et al., 2014; Kollars, 2017; Schafer et al., 2020).

Para pacientes com suspeita de dengue ou doença pelo vírus Zika, os testes de amplificação de ácido nucleico (NAATs) são o método preferido de diagnóstico, porque podem fornecer evidências confirmadas de infecção e distinguir o vírus específico (WHO, 2020). Os testes de imunoglobulina M (IgM) e de anticorpos neutralizantes também podem ser usados para identificar infecções pelo vírus Zika e pelo vírus da dengue, especialmente em pacientes que procuram um médico depois que o ácido nucleico viral é indetectável. (Bingham et al., 2016; Lanciotti et al., 2008; Paz-Bailey et al., 2018). 
No entanto, o teste de anticorpos para dengue e Zika é complicado devido à reatividade cruzada, o que pode impedir a determinação final de qual flavivírus é a causa da infecção recente de uma pessoa (Lindsey et al., 2018; Lorenz et al., 2020).

Usando esses métodos, os casos de dengue e Zika podem ser confirmados após o recebimento dos casos suspeitos. No entanto, diante da nova pandemia da Covid-19, a nova situação no Brasil pode ter mudado a eficácia do controle dos arbovírus. Enquanto o sistema de saúde e as organizações globais trabalham para conter a disseminação da nova patologia em questão, o problema permanece na capacidade dos programas de vigilância de doenças emergentes, reemergentes e concomitantes (como a dengue) que podem afetar gravemente o sistema de saúde pública (Lokida et al., 2020; RodriguezMorales et al., 2020).

Diante do exposto, o objetivo desse estudo foi descrever a situação epidemiológica relacionada à subnotificação dos casos de dengue e Zika no ano de 2020, devido aos esforços voltados, principalmente, para o controle da pandemia do novo coronavírus, à ausência de busca por assistência médica e receio da população. Acredita-se que o resultado do estudo possa impactar de forma positiva nas estratégias de busca e continuidade do cuidado à população exposta, a fim de favorecer um melhor atendimento, visando prevenção e promoção em saúde, garantindo os princípios de integralidade, universalidade e equidade do Sistema Único de Saúde no estado de Minas Gerais.

\section{Metodologia}

Trata-se de um estudo ecológico, descritivo. A população do estudo foi constituída por pessoas que morreram ou procuraram atendimento referente a suspeita de dengue ou Zika no período de 2019-2020, residentes do estado de Minas Gerais. Os dados foram obtidos por meio do Sistema de Informações de Agravos de Notificação (SINAN) e o Sistema de Informações sobre Mortalidade (SIM), que são disponibilizados pelo Departamento de Informática do Sistema Único de Saúde (DATASUS)/ Plataforma de Informações em Saúde (TABNET). O software PRISM 8.0 (GraphPad Software Inc., San Diego, CA, EUA) foi utilizado para análises descritiva das médias da incidência de casos notificados e óbitos.

Os dados coletados estão disponíveis na rede de internet, cedidos pelo Ministério da Saúde para livre consulta, conferindo domínio público e garantindo o sigilo dos seres humanos envolvidos, não necessita, portanto, da submissão ao Comitê de Ética em Pesquisa (Brasil, 2012).

\section{Resultados e Discussão}

A pandemia da Covid-19 desestruturou todo um sistema de saúde mundial e o organizou para contenção da nova entidade emergente (Nascimento et al., 2021). No estado de Minas Gerais, não foi diferente. Os Gráfico 1 e 2 mostram a descrição da incidência da dengue e do Zika nas semanas epidemiológicas 8 a 30 do ano de 2019 e 2020. 
Gráfico 1 - Incidência de casos notificados da dengue no estado de Minas Gerais, 2019-2020.

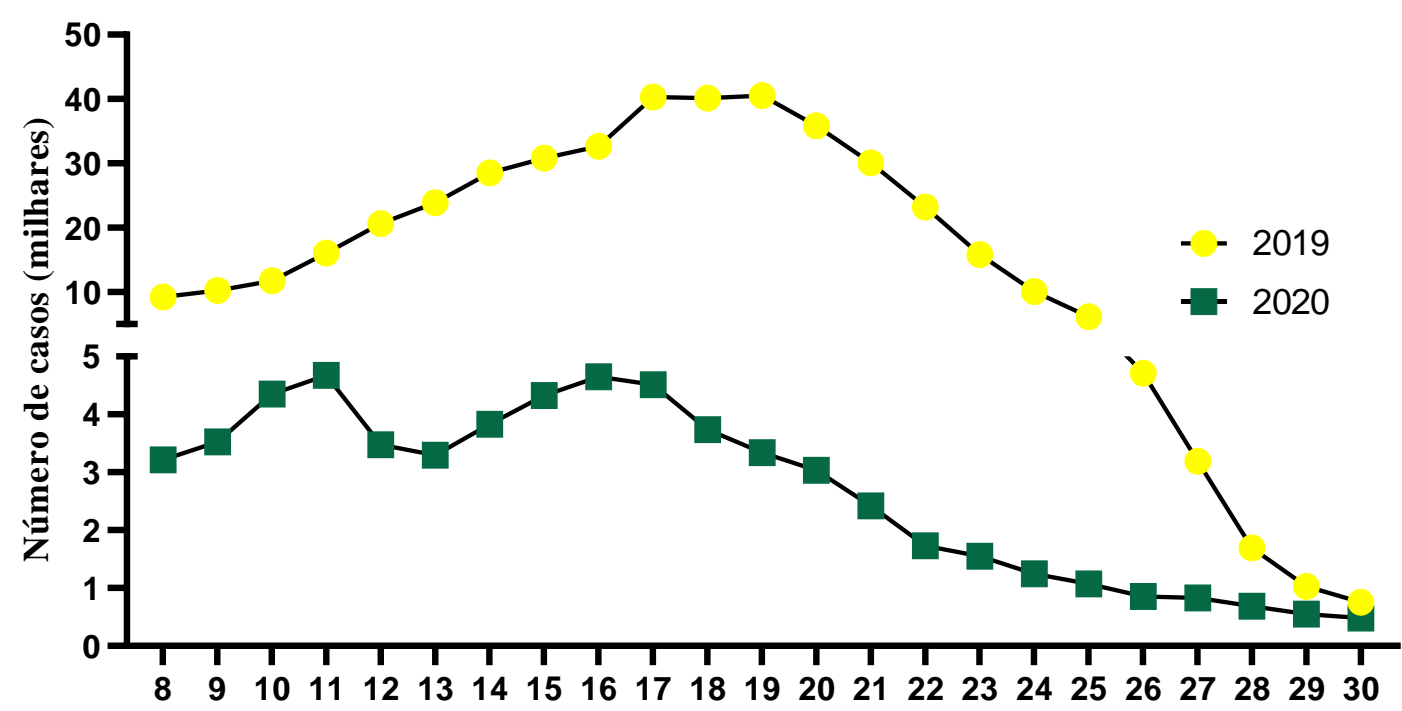

Semana Epidemiológica

Fonte: Autores.

Gráfico 2 - Incidência dos casos notificados de Zika no estado de Minas Gerais, 2019-2020.

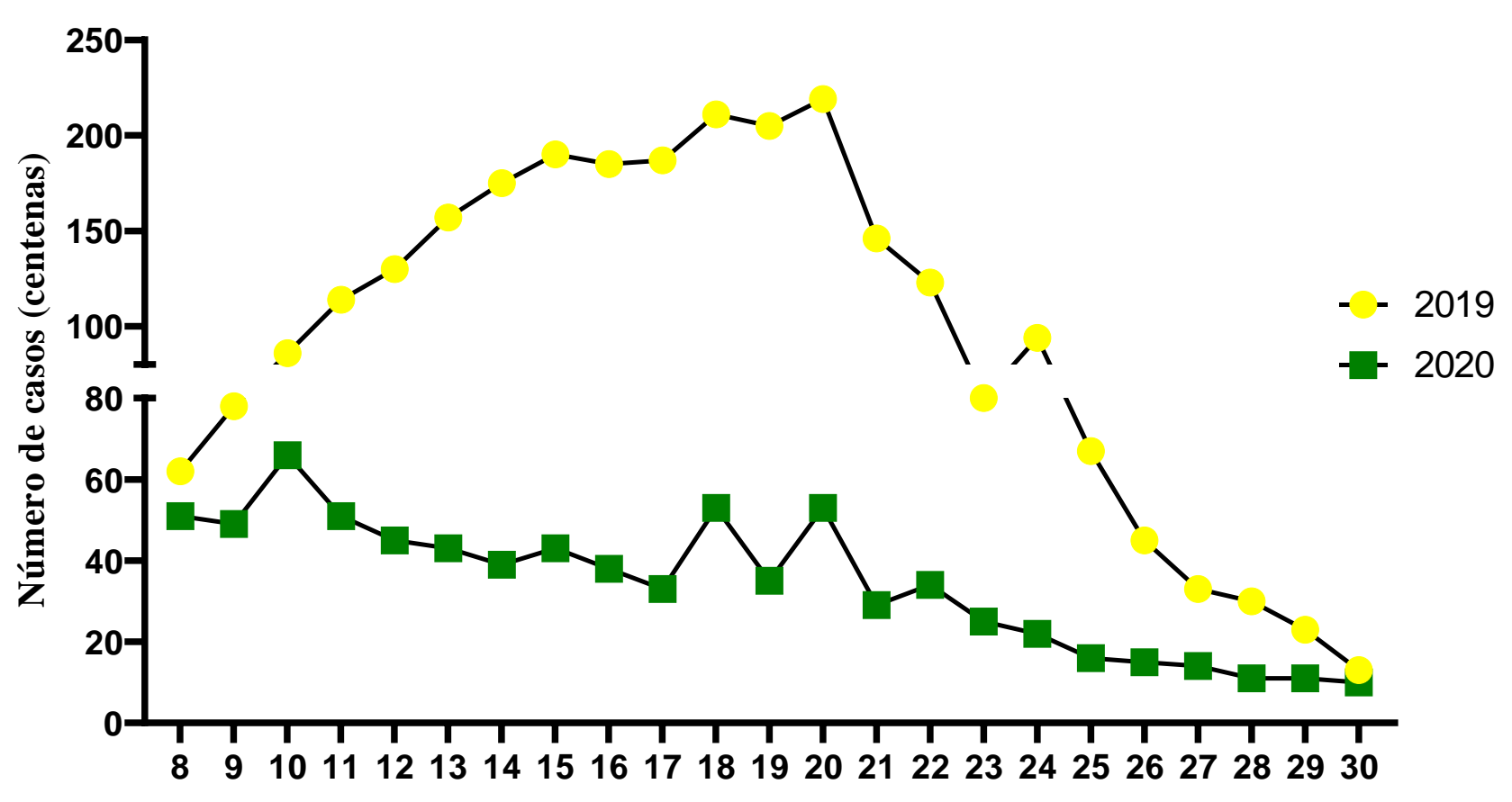

Semana Epidemiológica

Fonte: Autores.

Os dados revelam uma diminuição da incidência dos casos de dengue na semana epidemiológica 12, o mesmo se observa nos dados do Zika, data esta que marca o início dos casos notificados de Covid- 19. É observado ainda, que, nesse mesmo período em anos anteriores, exemplificado pelo a no de 2019, a dengue e o Zika teriam um aumento dos números de 
indivíduos acometidos, o que não foi obtido no ano de 2020. Achados semelhantes foram encontrados no estudo de (Leandro et al., 2020), que analisou o potencial impacto da pandemia da Covid-19 sobre as ações de controle da dengue e consequente redução em notificações no Brasil. Os resultados obtidos nesse estudo, mostraram uma mudança no comportamento da dengue em 2020 quando comparado aos anos anteriores, revelando uma diminuição no número de casos notificados a partir do período em que se iniciaram as ações contra a Covid-19.

No estado de Minas Gerias, no ano de 2020 foram confirmados apenas 85.356 casos de dengue e 1.350 de Zika, em 2019 esse número chegou a 478.898 casos de dengue e 3.228 nos casos de Zika, ou seja, houve uma redução de $82,17 \%$ na notificação em 2020 da dengue e de 58,17\% nos casos de Zika. Os óbitos por dengue no estado em 2019 foram 186 , enquanto no ano da pandemia foram apenas 14 como causa, redução de 92,47\%, em relação ao Zika não foram registrados em nenhum dos anos relacionados ao Zika vírus, mas 7 óbitos em 2019 e 2 óbitos em 2020 por outras causas, mas relacionadas ao mesmo.

Esses dados podem ressaltar três grandes questões: quanto ao receio da população na busca por auxílio médico e possível contaminação pelo novo agente, quanto a coinfecção dengue, Zika e Covid -19 e quanto a subnotificação dos casos (Nascimento, et al., 2021). Segundo (Ornell et al., 2020), a pandemia da Covid-19, também pode vista como a "pandemia de medo", uma vez que as informações dúbias ou mesmo falsas sobre fatores relacionados à transmissão do vírus, o período de incubação, seu alcance geográfico, o número de infectados e a taxa de mortalidade real levaram à insegurança e ao medo na população. Assim, embora a dengue e o Zika representem risco para a população, acredita-se que haja receio na busca de assistência médica por medo do risco de contaminação pela Covid-19 (Nascimento et al., 2021). Além disso, vale ressaltar que não urgentes de dengue e Zika podem ter sido postergados, por esse provável pânico de contaminação da população já citado. Quanto à coinfecção, acredita-se que tais patologias possam ocorrer simultaneamente ou de forma isolada, tornando o diagnóstico e o combate a tais doenças um desafio preocupante (Ridwan, 2020). Devido aos esforços voltados para a contenção do novo agente infeccioso e aos fatores supracitados relacionados ao medo da população e à possível coinfecção dengue, Zika, Covid-19, a notificação dos casos das arboviroses pode ter sido prejudicada.

Gráfico 3 - Incidência de dengue e Zika x Covid-19 no estado de Minas Gerais, em 2020.

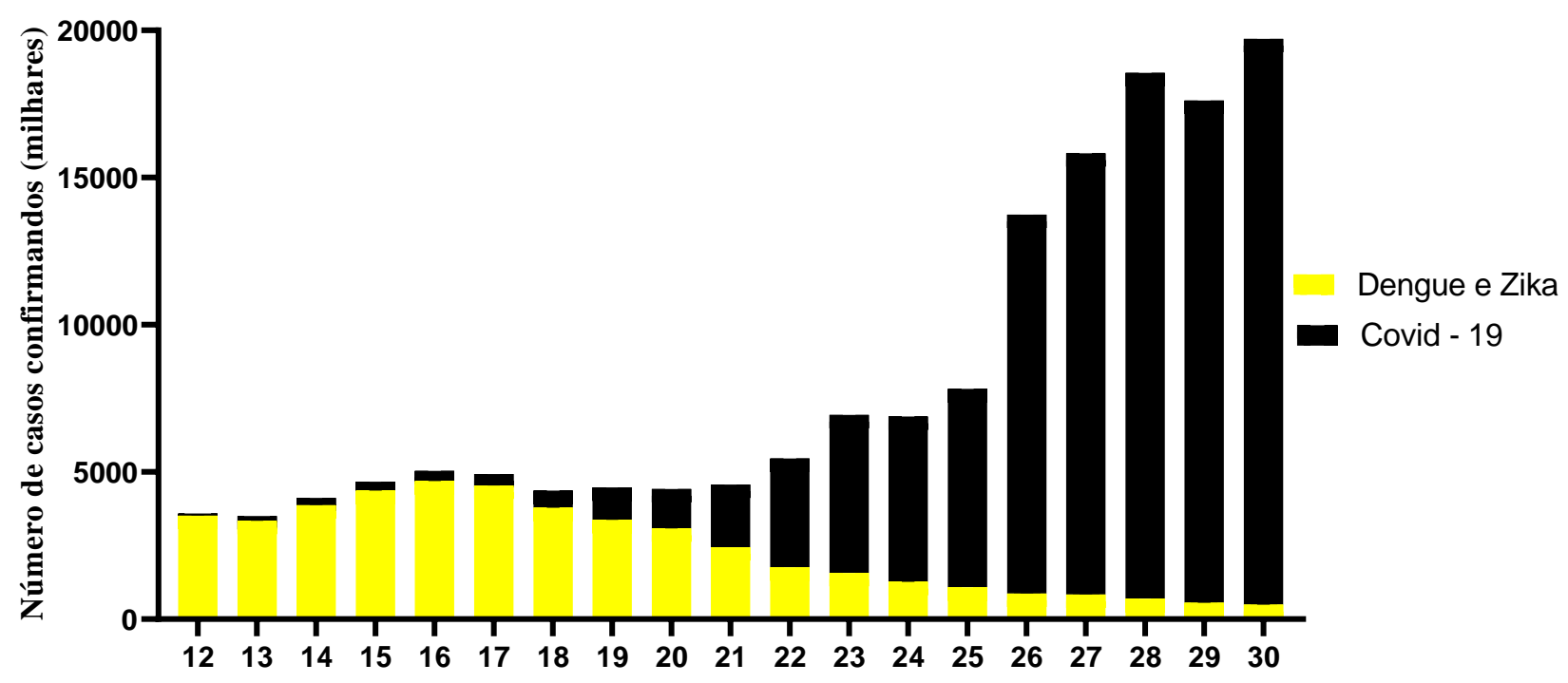

Semana Epidemiológica 
O Gráfico 3 mostra a incidência de dengue e Zika nas semanas epidemiológicas (SE) 12-30 do ano de 2020 e Covid 19 nas SE 12-30 de 2020. Embora existam poucos casos de dengue e Zika em comparação com a gama de casos de Covid19, é importante notar que o número de casos em 2019 ainda era considerável. O registro de casos notificados de Covid-19 em 2020 aumentou exponencialmente, enquanto o da dengue e Zika diminuíram. Esse gráfico reforça a ideia de que os esforços em resposta à pandemia da Covid-19 podem ter prejudicado a realização de estratégias eficientes para controle das arboviroses.

Acredita-se que com o avanço da Covid-19, em Minas Gerais, ocorreu redução do patrulhamento de criadouros de mosquitos nas residências, pelos agentes endêmicos.

As estatísticas indicam que os casos de dengue deveriam ter aumentado, confirmando a hipótese de subnotificação de casos. Estudos realizados em outros estados também mostram que há menos notificações de dengue em 2020 em Sergipe (Nascimento et al., 2021), que houve uma redução de 5164 casos de 2019 para 959 casos em 2020, redução de 81,42\% nas notificações, no Piauí (Mascarenhas et al., 2020) mostrou que houve redução de 74,5\% nos casos de notificação de dengue no ano de 2019 para 2020 e em São Paulo(Furtado \& Silveira, 2021) demostrou uma redução de 55,8\% na notificação dos casos. Além disso, estudos realizados em outros países, como a Colômbia, mostraram resultados similares, observando-se uma redução sazonal coincidente nas notificações de dengue com o aumento das notificações de Covid-19 (RodriguezMorales et al., 2020).

Os dados obtidos sugerem que esforços para conter o avanço da Covid-19 podem inviabilizar a notificação e o diagnóstico efetivo das citadas patologias. Além disso, sintomas semelhantes entre doenças também podem contribuir, ou pode ser um caso de coinfecção. Portanto, acredita-se que, como nenhum dos dois tem um tratamento específico e tende a se resolver por conta própria, um teste diagnóstico para dengue pode ser realizado, portanto, sem aviso prévio. Quanto à busca de atendimento médico específico, entende-se que o pânico entre as pessoas infectadas pela Covid-19 dificulta a notificação e retarda o diagnóstico por não procurarem ou procurarem com menor frequência.

As limitações do estudo são do viés ecológico que é sempre lembrado como uma limitação para o uso de correlações ecológicas.

\section{Conclusão}

A situação epidemiologica relacionada à subnotificação dos casos de dengue e Zika no ano de 2020 parece estar diretamente associada ao início da pandemia da Covid-19. Entre os fatores principais que levaram à redução do número de casos dessas arboviroses no ano de 2020, acredita-se que o receio da população na busca por auxílio médico em meio a pandemia, os possíveis casos de coinfecção dengue, Zika e Covid-19 e a consequente subnotificação dos casos contribuíram de forma relevante.

O tema desta pesquisa levanta questões que podem ser ampliadas e aprofundadas por novas pesquisas a fim de melhor compreender e refletir sobre os dados aqui coletados. Portanto, pode-se determinar a importância do acordo, principalmente em áreas endêmicas, para que medidas eficazes e mais rígidas de controle da doença possam ser adotadas. Sabe-se que tanto a dengue quanto o Zika podem se desenvolver para formas fatais, portanto, é importante o desenvolvimento de políticas que incentivem a notificação, o controle do patógeno, a educação da população e medidas de saúde em função do diagnóstico precoce e monitoramento ditadores necessários e preditivos. Para trabalhos futuros, recomenda-se realizar uma investigação aprofundada dos protocolos seguidos pelas agências reguladoras da dengue, Zika e Covid-19, e especificar melhor os motivos da redução nas notificações de casos suspeitos e confirmados. 
Research, Society and Development, v. 10, n. 16, e67101623207, 2021

\section{Referências}

Bingham, A. M., Cone, M., Mock, V., Heberlein-Larson, L., Stanek, D., Blackmore, C., \& Likos, A. (2016). Morbidity and Mortality Weekly Report Comparison of Test Results for Zika Virus RNA in Urine, Serum, and Saliva Specimens from Persons with Travel-Associated Zika Virus Disease - Florida, 2016. 65(18), 475-478.

Brasil, M. da S. (2012). Resolução No 466, DE 12 de dezembro de 2012 (Vol. 66, Issue December).

Eiras, A. E., Buhagiar, T. S., \& Ritchie, S. A. (2014). Development of the Gravid Aedes Trap for the Capture of Adult Female Container-Exploiting Mosquitoes (Diptera: Culicidae). Journal of Medical Entomology, 51(1), 200-209. https://doi.org/10.1603/ME13104

Furtado, . J. L. C., \& Silveira, . R. de C. V. da. (2021). Efeitos da pandemia em relação aos números de caso de dengue no estado de são paulo e no município de Jaú. Revista Multidisciplinar Em Saúde, 2, 11.

Kollars, T. M. (2017). Assessing likely invasion sites of Zika virus-infected mosquitoes in civilian and naval maritime ports in Florida. Research and Reports in Tropical Medicine, Volume 8, 1-6. https://doi.org/10.2147/rrtm.s123456

Lanciotti, R. S., Kosoy, O. L., Laven, J. J., Velez, J. O., Lambert, A. J., Johnson, A. J., Stanfield, S. M., \& Duffy, M. R. (2008). Genetic and serologic properties of Zika virus associated with an epidemic, Yap State, Micronesia, 2007. Emerging Infectious Diseases, 14(8), 1232-1239. https://doi.org/10.3201/eid1408.080287

Leandro, C. dos S., Barros, F. B. de, Cândido, E. L., \& Azevedo, F. R. de. (2020). Redução da incidência de dengue no Brasil em 2020: controle ou subnotificação de casos por COVID-19? Research, Society and Development, 9(11), e76891110442. https://doi.org/10.33448/rsd-v9i11.10442

Lindsey, N. P., Staples, J. E., Powell, K., Rabe, I. B., Fischer, M., Powers, A. M., Kosoy, O. I., Mossel, E. C., Munoz-Jordan, J. L., Beltran, M., Hancock, W. T., Toews, K. A. E., Ellis, E. M., Ellis, B. R., Panella, A. J., Basile, A. J., Calvert, A. E., Laven, J., Goodman, C. H., \& Hillsa, S. L. (2018). Ability to serologically confirm recent Zika virus infection in areas with varying past incidence of dengue virus infection in the United States and U.S. Territories in 2016. Journal of Clinical Microbiology, 56(1), 1-6. https://doi.org/10.1128/JCM.01115-17

Lokida, D., Lukman, N., Salim, G., Butar, D. P. B., Kosasih, H., Wulan, W. N., Naysilla, A. M., Djajady, Y., Sari, R. A., Arlinda, D., Lau, C. Y., \& Karyana, M. (2020). Diagnosis of COVID-19 in a Dengue-Endemic Area. American Journal of Tropical Medicine and Hygiene, 103(3), 1220-1222. https://doi.org/10.4269/ajtmh.20-0676

Lorenz, C., Azevedo, T. S., \& Chiaravalloti-Neto, F. (2020). COVID-19 and dengue fever: A dangerous combination for the health system in Brazil. Travel Medicine and Infectious Disease, 35(March), 101659. https://doi.org/10.1016/j.tmaid.2020.101659

Mascarenhas, M. D. M., De Araújo Batista, F. M., Rodrigues, M. T. P., De Alencar Alves Barbosa, O., \& Barros, V. C. (2020). Simultaneous occurrence of COVID-19 and dengue: what do the data show? Cadernos de Saude Publica, 36(6). https://doi.org/10.1590/0102-311x00126520

Nascimento, C. S., Correia, J. P. S., Temóteo, C. C. S., \& Campos, A. L. B. (2021). Impactos no perfil epidemiológico da Dengue em meio a Pandemia da COVID-19 em Sergipe. Research, Society and Development, 10(5), e3610514544. https://doi.org/10.33448/rsd-v10i5.14544

Ornell, F., Halpern, S. C., Paim Kessler, F. H., \& de Magalhães Narvaez, J. C. (2020). The impact of the COVID-19 pandemic on the mental health of healthcare professionals. Cadernos de Saude Publica, 36(4). https://doi.org/10.1590/0102-311X00063520

Paz-Bailey, G., Santiago, G. A., Klein, L., \& Perez-padilla, J. (2018). Persistence of Zika Virus in Body Fluids — Preliminary Report. N Engl J Med, 379(13), 1234-1243. https://doi.org/10.1056/NEJMoa1613108.Persistence

Pereira, J. O., Carvalho, J. T., Marçal, D., Vimercate, L., Gomes, C., \& De, D. S. (2019). Situação Epidemiológica da Dengue , Chikungunya e Zika , no Brasil , em Minas Gerais e no Espírito Santo . e sanitárias, diante às constantes epidemias nas últimas décadas e às transformações. $2018-2019$.

Ridwan, R. (2020). COVID-19 and dengue: a deadly duo. Tropical Doctor, 50(3), 270-272. https://doi.org/10.1177/0049475520936874

Rodriguez-Morales, A. J., Cardona-Ospina, J. A., \& Gutiérrez-Ocampo, E. (2020). Clinical, laboratory, and imaging features of pediatric COVID-19: A systematic review and meta-analysis. Travel Medicine and Infectious Disease, 100(15), e25230. https://doi.org/10.1097/MD.0000000000025230

Schafer, M. S., Claros, C. B. de O., Damasceno, V. Y. P., Penteado, M. C., Dourado, T. F., \& Costa, R. S. L. da. (2020). Perfil epidemiológico da Covid-19 no Estado do Paraná Epidemiological profile of Covid-19 in the State of Paraná. Research, Society and Development, 10(13), 39-61. https://orcid.org/0000-00031754-9238.

Sharp, T. M., Fischer, M., Muñoz-Jordán, J. L., Paz-Bailey, G., Staples, J. E., Gregory, C. J., \& Waterman, S. H. (2019). Dengue and Zika Virus Diagnostic Testing for Patients with a Clinically Compatible Illness and Risk for Infection with Both Viruses. Recommendations and Reports, 68(1).

WHO, W. H. O. (2020). Diagnostic testing for headache. Medical Clinics of North America, 85(4), 865-885. https://doi.org/10.1016/S0025-7125(05)70348-5 